\title{
Antiparticle to particle production ratios in hadron-hadron and $d$-Au collisions in the DPMJET-III Monte Carlo model
}

\author{
F. W. Bopp* and J. Ranft ${ }^{\dagger}$ \\ Fachbereich Physik, Universität Siegen, D-57068 Siegen, Germany \\ R. Engel ${ }^{\ddagger}$ \\ Forschungszentrum Karlsruhe, Institut für Kernphysik, Postfach 3640, D-76021 Karlsruhe, Germany \\ S. Roesler ${ }^{\S}$ \\ CERN, Geneva, Switzerland \\ (Received 13 July 2007; published 18 January 2008)
}

\begin{abstract}
To understand baryon stopping we analyze new Relativistic Heavy Ion Collider and Fermilab data within the framework of the multichain Monte Carlo DPMJET-III. The present consideration is restricted to hadron-hadron and $d$-Au collisions, where the present version of the model can be trusted.
\end{abstract}

DOI: 10.1103/PhysRevC.77.014904

PACS number(s): 13.85.Ni, 13.85.Hd, 13.60.Rj, 25.75.Dw

\section{INTRODUCTION}

The theoretical tools available at present are not sufficient to understand hadronic collisions at high energies from quantum chromodynamics (QCD) alone. Phenomenological models of so-called soft multiparticle production are typically applied in addition to perturbative QCD. The dual parton model (DPM) [1] is such a phenomenological model. It is quite successful in presenting many details in the multiparticle production. Its fundamental ideas are presently the basis of many of the Monte Carlo implementations of soft interactions.

The properties of our DPM implementation DPMJET-III are described in Refs. [2-6]. For a more detailed description of DPMJET-III we refer to these articles and the literature quoted therein.

A feature of hadron production in nuclear collisions discussed in the past 10 years is the large stopping of the participating nucleons in hadron-nucleus and nucleus-nucleus collisions. Experimental data were presented in Refs. [7-9] and [10] that clearly demonstrate the sizable stopping of the participating nucleons in hadron-nucleus and nucleus-nucleus interactions for fixed-target experiments.

Multistring fragmentation models like DPM or similar models contain some stopping, but in their original form they did not account for the enhanced stopping found in nuclear collisions. Therefore, to incorporate the effect into multistring fragmentation models new diquark breaking DPM diagrams acting in hadron-nucleus and nucleus-nucleus collisions were proposed by Refs. [11] and [12] and investigated in detail in Refs. [13] and [14]. Similar ideas were discussed by Refs. [15] and [16]. The Monte Carlo implementation into DPMJET-II.5 of the new diquark breaking diagrams of Refs. [11] and [12] was discussed in Ref. [17]. The implementation into DPMJET-III [6]

\footnotetext{
*bopp@physik.uni-siegen.de

†Johannes.Ranft@cern.ch

†Ralph.Engel@ik.fzk.de

§Stefan.Roesler@cern.ch
}

of these diagrams differs somewhat from that of Ref. [17] and was described in Refs. [18,19].

A second mechanism, which can contribute to baryon stopping in nuclear collisions occurs during the fusion of chains $[20,21]$. This mechanism was introduced into DPMJET-III to accommodate the Relativistic Heavy Ion Collider (RHIC) data [22,23]. We will use chain fusion here exactly as described in these articles.

Particle production ratios in $p-p$ and $d$-Au collisions measured at RHIC [24-27] allow a more precise determination of the parameters of anomalous baryon stopping. The needed stopping contribution is described in Sec. II. Sections III and IV consider the available data on stopping for proton-proton and deuteron-gold reactions.

In Sec. V we consider hyperon productions ratios in pionproton collisions. One aspect of these data again require a minor adjustment.

\section{IMPLEMENTATION OF NEW DIAGRAMS FOR AN IMPROVED DESCRIPTION OF BARYON STOPPING}

As justified by Rossi and Veneziano [28] in the framework of a $1 / N$ expansion a baryon can be pictured as made of three quarks bound together by three strings that join in a socalled string junction point. In diagrams one can characterize the baryons (i) by the three quarks and the string junction or (ii) by a quark and a diquark. In the second case the string junction always goes with the diquark.

Figures 1-4 are quark line diagrams describing the production chains of particles. In these diagrams quarks and diquarks are usually plotted as single respectively paired solid lines. If diquarks break, string junctions are added as dashed lines.

In the fragmentation of diquarks for the first fragmentation step usually two possibilities are considered. Either one gets a baryon, which contains the diquark (with the string junction), or one gets a meson containing only one of the valence quarks. In this case the string junction migrates to the next step. This 


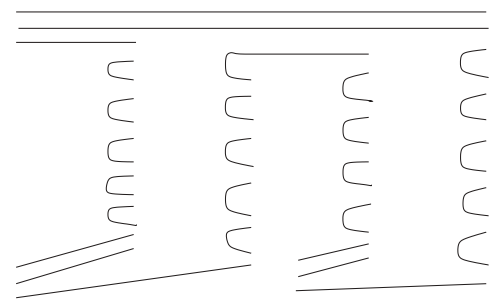

FIG. 1. The diquark-conserving diagram for a nucleon-nucleus collision with two participants of the target nucleus.

can continue and the baryon is eventually produced in one of the following fragmentation steps.

This migration mechanism is well known; it is presented in the review of the DPM [1] and it was investigated in, for instance, Refs. [29,30]. Actually it was implemented in the BAMJET fragmentation code $[31,32]$ used in the early versions of DPMJET a long time ago (1980). The mechanism is also implemented under the name popcorn fragmentation in the Lund chain fragmentation model JETSET/PYTHIA $[33,34]$ that is presently used in DPMJET-III.

What happens in the model with the popcorn mechanism compared to the model without it can be most easily seen by looking at the proton rapidity distribution in $p$ - $p$ collisions. With the mechanism the two maxima of the proton rapidity distribution in the target and projectile fragmentation region shift by about half a unit to the center; moreover, the peaks become wider and correspondingly the dip in the center reduces. At the same time the Feynman $x$ distributions of mesons get a component at larger Feynman $x$. The effects in hadron-nucleus and nucleus-nucleus collisions are quite similar.

However, the popcorn mechanism alone cannot explain the baryon stopping observed experimentally in hadron-nucleus and nucleus-nucleus collisions $[7,8]$.

\section{A. Nuclear diquark breaking}

Most interesting for DPMJET-III is the so-called second $C$ - $K$ mechanism [12-14,35]. In Fig. 1 we first plot the diquarkconserving diagram for a nucleon-nucleus collisions with two participants of the target nucleus. This is the traditional way for such a collision in the DPM. The Glauber rescattering forced the introduction of Glauber sea quarks at the top

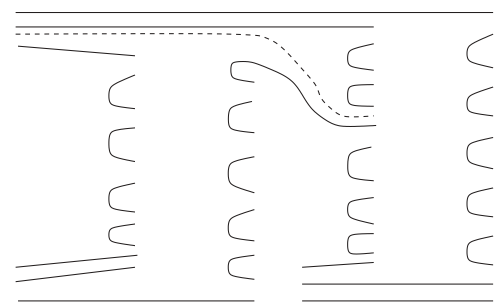

FIG. 2. The Glauber sea quark mechanism of baryon stopping (denoted as GSQBS) for a nucleon-nucleus collision with two participants of the target nucleus. ends of the inner fragmentaion chains. In Fig. 2 we then plot the second $\mathrm{C}-\mathrm{K}$ diquark-breaking diagram for the same collision. Now a Glauber sea quark from the first interaction attaches to the string junction. This breaks the diquarks. As it involves Glauber quarks, we will call the mechanism the Glauber sea quark mechanism of baryon stopping (GSQBS). The probability of such a diquark splitting rises with the size of the nuclei as the scattering then involves more and more such interactions.

A new type of string combination appears in the second interaction, which spans one of the original valence diquark quarks (the top one), the junction line, and a valence quark at the bottom. The baryon ends up at the position of the junction line. The central assumption is now that the sea quark of the initial scattering determines this position. One of the valence quarks that its initial momentum connects to the junction line by an upward string. In this way the string pulling the vortex line down is compensated by a string pulling it up.

The GSQBS picture is chosen for simplicity. Formally at the very top three strings and a vortex line are exchanged. In the topological view of the total scattering amplitude it is a cut $t$-channel quarkless baryonium exchange. Usually baryonium exchanges have a rather low intercept and the idea is that in special situations a small quarkless component with a high intercept appears. By taking the position from a Glauber sea quark a flattish distribution of the vortex line with a intercept of $1 / 2$ is implied, with an added bias to larger values as the sea quark actually had to sit on the forward end of string of a minimum size. In the factorizing version of the model [36] this bias does not exist. The intercept is then estimated to be slightly less than 1 .

The GSQBS has been implemented into DPMJET-II.5 and DPMJET-III. With this mechanism one is able to fill the dip in the baryon rapidity distributions at central rapidity in agreement with the experimental data. The mechanism also contributes to the increase of hyperon production in nucleon-nucleus and nucleus-nucleus collisions. This was discussed in some detail in Refs. [14,35].

\section{B. Hadronic diquark breaking at high energy}

At high energies multiple collisions appear even in hadronhadron scattering due to the unitarization procedure. This has the consequence that new diagrams, such as the GSQBS diagram, become necessary. In some way or other such diagrams have to be implemented in any model that includes both elastic and inelastic processes.

We call the sea quarks at the ends of the additional chains in this case unitary sea quarks. They are relevant at high energies. The Glauber sea quarks are needed in nuclear collisions at rather low energies, for instance, at the energies of heavy-ion collisions at the CERN-SPS. In contrast to this, unitary sea quarks were found to appear in significant numbers only at rather high energies, for instance, at the energies of RHIC, the CERN-SPS collider, or the Tevatron collider.

With the unitary sea quarks at the ends of the chains from the secondary collisions one obtains a new mechanism for baryon 


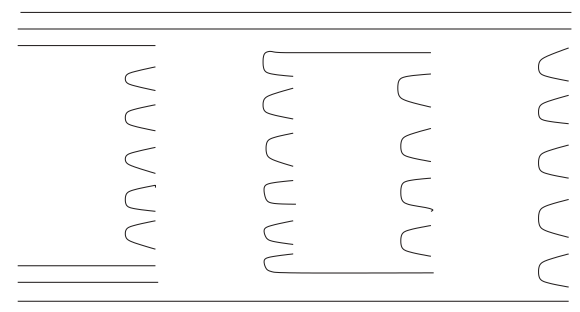

FIG. 3. Standard DPM diagram for a nucleon-nucleon interaction with one additional soft secondary interaction induced by the unitarization procedure.

stopping that will become effective at very high energies. It is illustrated in Figs. 3 and 4.

The standard DPM diagram is plotted in Fig. 3 for a nucleon-nucleon interaction with two soft interactions induced by the unitarization procedure. As in Fig. 1, there is one valence-valence and one sea-sea interaction, each represented by a pair of chains. In analogy to Fig. 2, a new diagram [17] for baryon stopping is constructed in Fig. 4. The diquark is split and a unitary sea quark is used to have the baryon only in the second or later fragmentation steps in one of the chains. We call this the unitary sea quark mechanism for baryon-stopping USQBS. The implementation of the new diquark breaking diagrams in DPMJET-III is discussed in detail in contributions $[18,19]$ to conferences. The relative probability of this process introduces one parameter, which will be given later.

Again the probability for such a diquark splitting rises if there are more than two interactions of the hadrons involved. Obviously, this mechanism leads to Feynman $x$ distributions of baryons in $p$ - $p$ collisions becoming softer and Feynman $x$ distributions of mesons becoming harder than without the USQBS mechanism.

In contrast to the GSQBS mechanism that leads to observable changes in nuclear collisions at the energy of the CERN-SPS, there were no data available before RHIC to prove that this USQBS mechanism is a needed extension of the DPM. The situation concerning baryon stopping in proton-proton or antiproton-proton collisions at lower energies was inconclusive [37]. An idea to observe baryon stopping in diffractive events [38], which always contain two interactions, was unfortunately not pursued in experimental analysis.

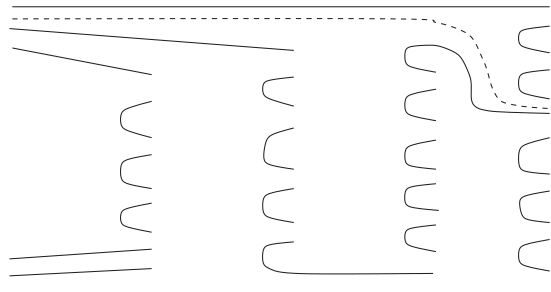

FIG. 4. New DPM diagram for a nucleon-nucleon interaction with one additional soft secondary interaction induced by the unitarization procedure. We call this unitary sea quark mechanism for baryon-stopping USQBS [17].

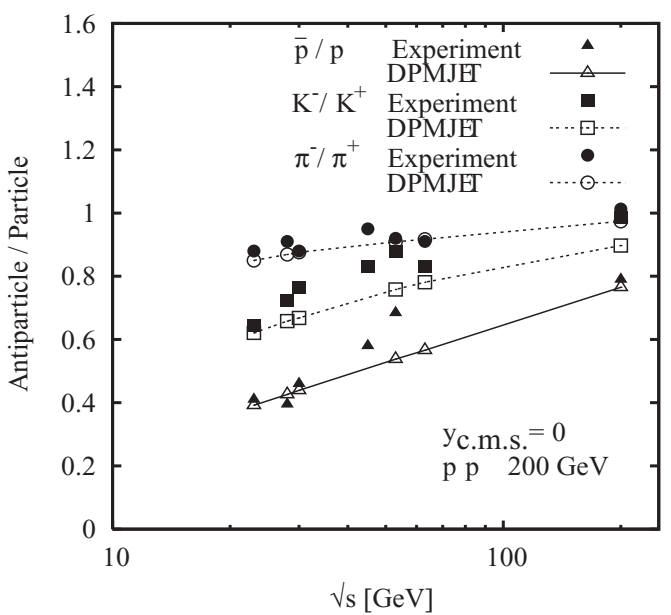

FIG. 5. $\sqrt{s}$ dependence of antiparticle-to-particle ratios in $p$ - $p$ collisions at $y_{\text {c.m. }}=0$. Experimental data are from the BRAHMS Collaboration at RHIC [27], the ISR [39], and the NA27 Collaboration [40]. We compare DPMJET-III results with the experimental data.

\section{New parameters connected with the diquark breaking diagrams}

For each of the new diquark breaking diagrams described in this section a new parameter has to be introduced. These parameters give the probability for the diquark breaking mechanisms to occur, given a suitable sea quark is available and given that the diquark breaking mechanism is kinematically allowed. For an original diquark-quark chain of small invariant mass, which originally just fragments into two hadrons, the diquark breaking is often not allowed at small energies.

The values of the new parameters are determined below by comparing DPMJET-III with experimental data on antiparticleto-particle production ratios and on net-baryon distributions. The fusion contribution that sometimes also involves baryon transport is kept as in previous fits [6].

\section{ANTIPARTICLE TO PARTICLE RATIOS IN PROTON-PROTON COLLISIONS}

With antibaryon to baryon ratios measured at RHIC one is now for the first time in a position to determine the USQBS parameter with good accuracy. In $p$ - $p$ collisions, the contribution of chain fusion is not very large.

Figure 5 compares measured $\pi^{-} / \pi^{+}, K^{-} / K^{+}$, and $\bar{p} / p$ ratios at $y_{\mathrm{c} . \mathrm{m} .}=0$ as function of the energy with the DPMJET-III results; of course, the USQBS mechanism influences essentially only the antiproton to proton ratio but also the comparison of the antimeson to meson ratios is of interest.

We get agreement to the data for $\pi^{-} / \pi^{+}$and $K^{-} / K^{+}$. To obtain agreement with the $\bar{p} / p$ ratio a USQBS parameter of. 07 had to be chosen to get a 0.1 reduction at the highest energy. It means that in $7 \%$ of the possible cases one transforms the diquark conserving diagram as given in Fig. 3 into the diquark breaking diagram given in Fig. 4. It corresponds to the parameter $\epsilon$ of $[36,41]$ whose stability against variation in the model was investigated in the cited articles. 

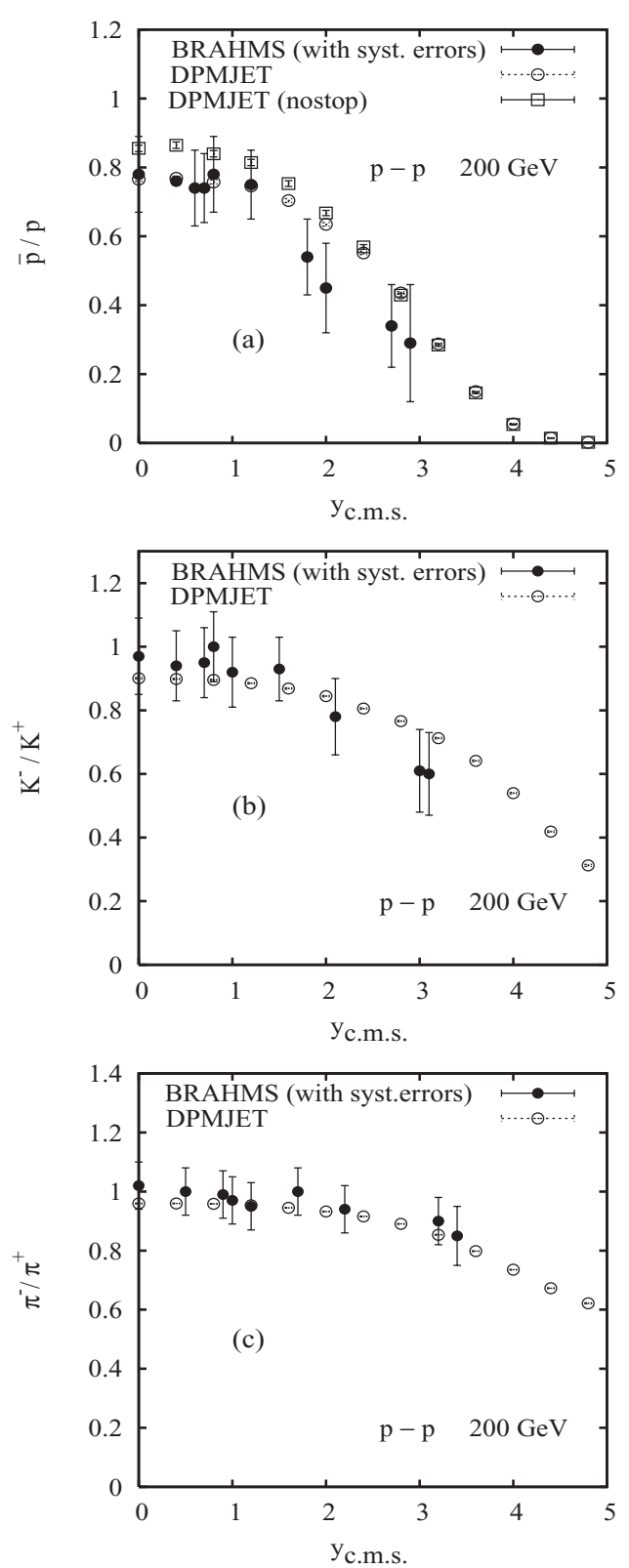

FIG. 6. Antiparticle-to-particle ratios, i.e., $\bar{p} / p$ (a), $K^{-} / K^{+}$(b), and $\pi^{-} / \pi^{+}$(c) in $p$ - $p$ collisions at $200 \mathrm{GeV}$ as function of the c.m. rapidity $y_{\mathrm{c} . \mathrm{m}}$. We compare data from the BRAHMS Collaboration at RHIC [27] (including the systematic errors as given in the BRAHMS article) with the results obtained from DPMJET-III. We plot the $\bar{p} / p$ ratios for the full DPMJET model as well as for the model without the USQBS diagrams ("nostop”).

The dependence of the three antiparticle-to-particle production ratios on the center-of-mass (c.m.) rapidity of the produced particles is plotted in Fig. 6. In this comparison of DPMJET-III with the BRAHMS data from RHIC [27] we have kept the USQBS parameters as determined above. The data are plotted with statistical and systematic errors as given in Ref. [27]. For clarification we compare in Fig. 6(a) the BRAHMS data for $\bar{p} / p$ ratios with the full DPMJET model as well as with the model without the USQBS diagrams. It shows that the USQBS diagrams are needed to get a better agreement with

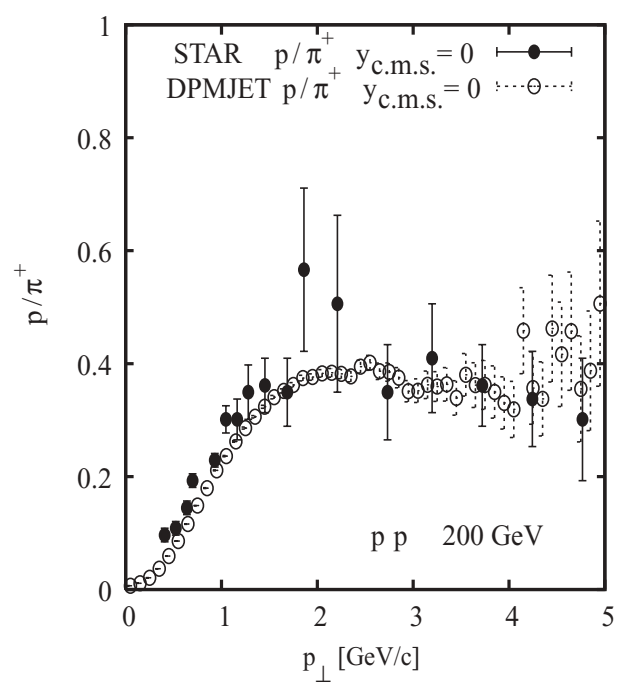

FIG. 7. The $p / \pi^{+}$particle production ratio as function of the transverse momentum in $\sqrt{s}=200 \mathrm{GeV}$ proton-proton collisions. We compare the ratio according to DPMJET-III with data from the STAR Collaboration [42].

the BRAHMS data at central rapidity. In all cases DPMJET-III describes the experimental data practically within the errors.

In Fig. 7 we plot the $p / \pi^{+}$particle production ratio as function of the transverse momentum in $\sqrt{s}=200 \mathrm{GeV}$ proton-proton collisions. The particle production ratio according to DPMJET-III is compared with data from the STAR Collaboration [42]. We find an excellent agreement.

\section{ANTIPARTICLE-TO-PARTICLE RATIOS IN $d$-AU COLLISIONS}

In $d$-Au collisions we have, in addition to the baryonstopping mechanisms acting in $p$ - $p$ collisions, the GSQBS diagrams and the contribution from chain fusion to baryon stopping.

The centrality dependence of charged antiparticle-toparticle ratios near midrapidity was measured by the PHOBOS Collaboration [24] in $d$-Au collisions at $\sqrt{s}=200 \mathrm{GeV}$ as a function of the centrality. The $\pi^{-} / \pi^{+}$and $\bar{p} / p$ ratios are compared in Fig. 8 with the DPMJET-III results. In this comparison the $\pi^{-} / \pi^{+}$and $\bar{p} / p$ ratios at all centralities agree within the experimental errors; the statistical errors of the Monte Carlo calculations are below \pm 0.02 .

Regarding the exact position of the points, the measurements and also our DPMJET-III calculations were done for four different centrality bins $(0-10 \%, 10-30 \%, 30-60 \%$, and 60-100\%) but the resulting antiparticle-to-particle ratios are plotted as function of $v$, the average number of collisions per deuterium participants. PHOBOS finds for the four centralities given above $v=8.1,6.1,4.0$, and 2.2 , in the DPMJET-III calculations we find $v=7.77,6.49,4.18$, and 2.14.

In Fig. 9 we plot the $\bar{p} / p$ and $\pi^{-} / \pi^{+}$ratios in $d$-Au collisions at $\sqrt{s}=200 \mathrm{GeV}$ as function of the transverse momentum. Compared are experimental data of the PHOBOS 


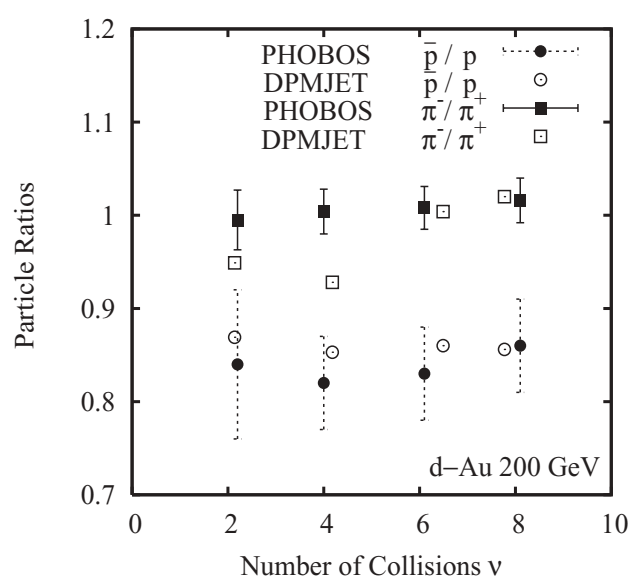

FIG. 8. Centrality dependence of charged antiparticle-to-particle ratios near midrapidity as measured by the PHOBOS Collaboration [25] in $d$-Au collisions at $\sqrt{s}=200 \mathrm{GeV}$ is compared to the DPMJET-III model.

and STAR Collaboration [25,43] with the results from DPMJET-III; we find a reasonable agreement.

In Figs. 10 and 11 we plot $\bar{\Lambda} / \Lambda$ ratios and net- $\Lambda$ production $(\Lambda-\bar{\Lambda})$ in $d$-Au collisions at $\sqrt{s}=200 \mathrm{GeV}$ as function of the center-of-mass rapidity. Compared are the experimental data of the STAR Collaboration [26] with the results of the DPMJET-III model. The agreement for the ratios $\bar{\Lambda} / \Lambda$ is satisfactory given the different binning; significant might be some disagreement for net $\Lambda$ production in the Au fragmentation region.

For all DPMJET-III calculations in Figs. 8 to 11 we use DPMJET-III with chain fusion as described in detail in Refs. [6,22,23]. The USQBS parameter is used exactly as described in the previous section. The GSQBS parameter was fitted. It turned out to be rather small at this energy and it was actually set to zero in the shown calculations.

The vanishing of the GSQBS contribution should be taken with care. As said above in nuclear collision there are two baryon-stopping mechanisms. In addition to the GSQBS mechanism baryon stopping is obtained as a side effect from chain fusion (e.g., when a $q q-q$ chain and a $\bar{q}-q$

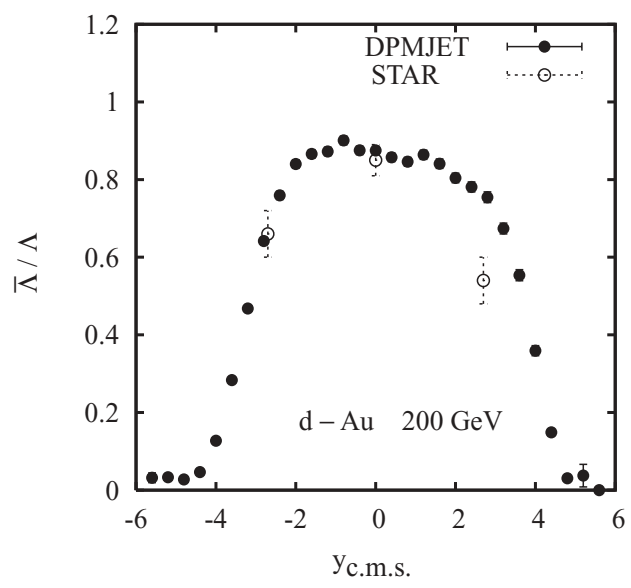

FIG. 10. Anti- $\Lambda$-to- $\Lambda$ ratio as function of the center-of-mass rapidity in minimum bias $d$-Au collisions at $\sqrt{s}=200 \mathrm{GeV}$. We compare the experimental data from the STAR Collaboration at RHIC [26] to the results from the DPMJET-III model.

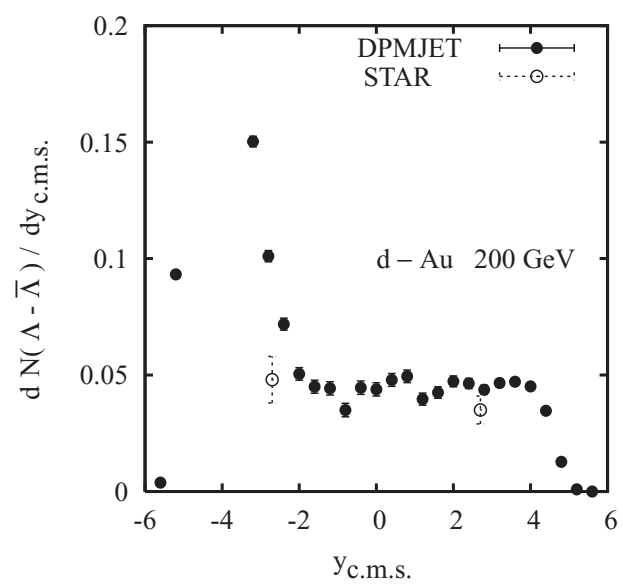

FIG. 11. Net- $\Lambda$ production as function of the center-of-mass rapidity in minimum bias $d$-Au collisions at $\sqrt{s}=200 \mathrm{GeV}$. We compare the experimental data from he STAR Collaboration at RHIC [26] to the results from the DPMJET-III model.
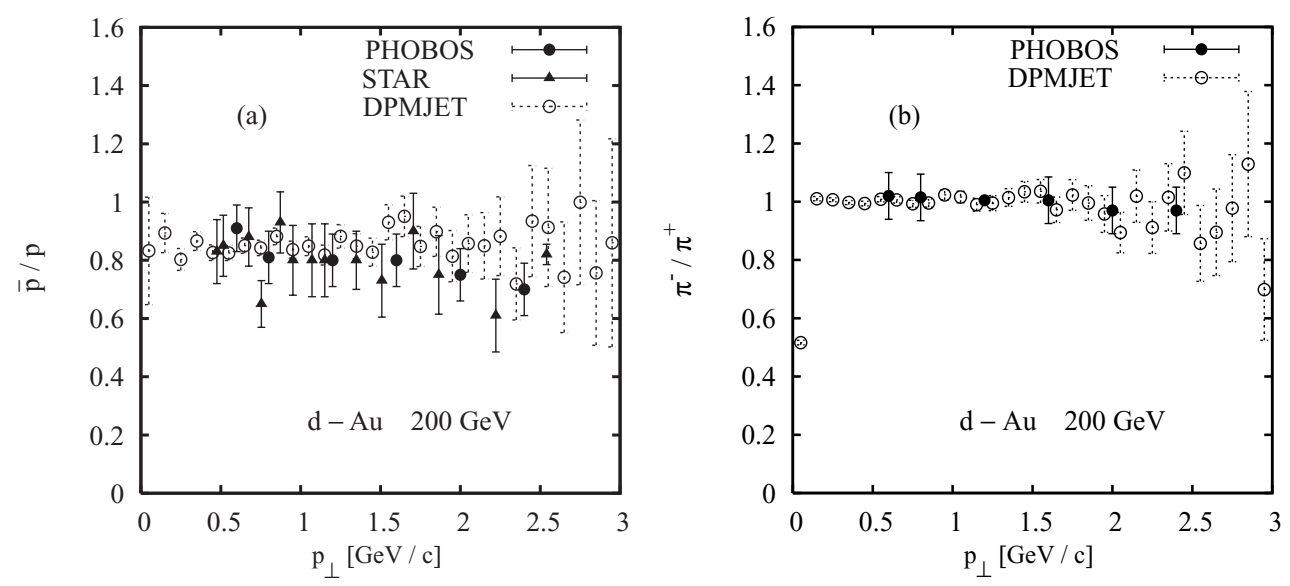

FIG. 9. $\pi^{-}$to $\pi^{+}$(b) ratios in $d$-Au collisions at $\sqrt{s}=200 \mathrm{GeV}$. We compare the data from the PHOBOS and the STAR Collaboration at RHIC $[25,43]$ to the results from the DPMJET-III model. 


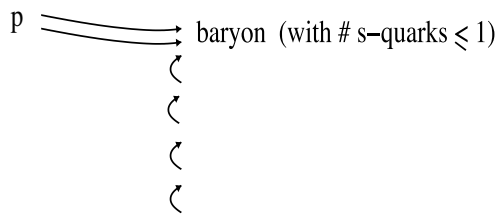

FIG. 12. Fragmentation into a baryon, the most important term in the fragmentation of a diquark.

chain fuse to a $q-q q$ chain) [22,23]. Both the intricate string structure of GSQBS and the forward-constituents-backwardconstituents structure of fusion strings are somewhat ad hoc and slight variations might lead to a redistribution between both contributions. More important is the fact that the observed nuclear baryon stopping is within the expected range (see also Ref. [44]).

\section{ANTIHYPERON-TO-HYPERON RATIOS IN HADRON-HADRON COLLISIONS}

Asymmetries of strange baryon production in $500 \mathrm{GeV}$ $\pi^{-}-p$ collisions were measured by the E791 Collaboration at Fermilab [9]. The asymmetry is defined as follows:

$$
A\left(\frac{B}{\bar{B}}\right)=\frac{N_{B}-N_{\bar{B}}}{N_{B}+N_{\bar{B}}}
$$

in each $x_{F}$ bin. These asymmeties are obviously closely related to the particle production ratios $\bar{B} / B$.

All measured asymmeties [9] $A(\Lambda / \bar{\Lambda}), A\left(\Xi^{-} / \bar{\Xi}^{+}\right)$, and $A\left(\Omega^{-} / \bar{\Omega}^{+}\right)$are positive, correspondingly in the given $x_{F}$ range the ratios $\bar{B} / B$ are smaller than 1 , there are more hyperons produced than antihyperons.

It was already noted by the E791 Collaboration in Ref. [9] that the JETSET/PYTHIA code $[33,34]$ gives $A\left(\Omega^{-} / \bar{\Omega}^{+}\right)$and $A\left(\Xi^{-} / \bar{\Xi}^{+}\right)$asymmetries, which are negative or zero in part of the kinematic range, correspondingly in part of the kinematic range the JETSET/PYTHIA code predicts more double or triple strange antihyperons than hyperons. This observation was also discussed in detail by Liu et al. [45].

DPMJET-III uses JETSET/PYTHIA for the fragmentation of the hadronic strings, the building blocks of the model. Therefore, we are not surprised that also the original DPMJET-III gives $A\left(\Omega^{-} / \bar{\Omega}^{+}\right)$and $A\left(\Xi^{-} / \bar{\Xi}^{+}\right)$asymmetries, which are negative or zero in part of the kinematic range in contrast to the experimental data.

To find the reason for this wrong behavior of chain fragmentation models like DPMJET-III or PYTHIA we have to consider the mechanism for baryon (and especially doubleand triple-strange hyperon) production in chain decay models.

In Fig. 12 we plot the standard diagram of chain decay models for the fragmentation of a diquark into a leading baryon. In collisions of nonstrange hadrons the chain-end diquarks do not contain strange quarks; therefore only hyperons with one strange quark can be produced this way. These are the $\Lambda$ hyperons in the E791 experiment.

Next, in Fig. 13 we plot the diagram for the fragmentation of our chain into a nonleading antibaryon-baryon pair. The antibaryon and baryon contain no quarks from the original

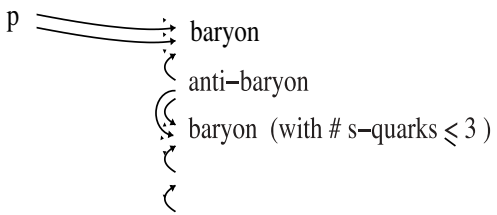

FIG. 13. Production of a antibaryon-baryon pair in the standard fragmentation of a diquark.

hadrons involved in the collision. Therefore, each of the quark or antiquarks involved can be strange; the antibaryon and the baryon can be double-strange or even triple-strange hyperons, for instance, $\bar{\Xi}, \Xi, \bar{\Omega}$, or $\Omega$ hyperons. But we note according to this mechanism the fragmentation into doublestrange or triple-strange antihyperons is favored against the fragmentation into double-strange or triple-strange hyperons. This diagram (or slight variations of it) is the only diagram available in the JETSET/PYTHIA chain fragmentation to produce antihyperon-hyperon pairs in diquark fragmentation. This explains the wrong behavior of PYTHIA and DPMJET-III discussed above.

To correct the behavior of DPMJET-III we have to add one missing diagram in diquark fragmentation. We did not correct ${ }^{1}$ the PYTHIA program, but we added the missing fragmentation step in DPMJET-III code before it calls the PYTHIA routines.

The missing diagram: We note that in the PYTHIA chain fragmentation diquark-antidiquark pairs can be exchanged in any position (see Fig. 13) except near to the chain-end diquarks. In Fig. 14 we plot the missing diagram. At the chain end we obtain a baryonium-like diquark-antidiquark pair; it has to fragment into a pair of mesons. Next to this in the chain we obtain a baryon. All three quarks of his baryon can be strange quarks. Therefore, in this diagram we can obtain double-strange and triple-strange hyperons, which dominate against the antihyperons produced eventually later in the chain fragmentation. Obviously, this is the missing mechanism needed in DPMJET-III.

To introduce the mechanism according to Fig. 14 we have to introduce a new parameter, which describes the probability that the new diagram is to be used in the first diquark fragmentation step. We obtain good results with the rather small probability of such a baryonium of $B_{2 \text { Mesons }}=0.01$. For the flavor distribution of this baryonium and the exchanged diquark-antidiquark pair we use a rather small value for the strangeness probability of $r_{s}=5 \%$.

${ }^{1}$ This has the advantage that we continue to use the standard PYTHIA
and that we therefore will be able to replace PYTHIA with new versions.

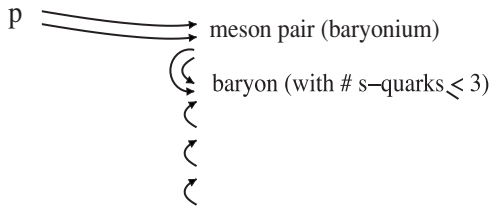

FIG. 14. Nonstandard fragmentation of a diquark into a leading meson pair and a baryon. 


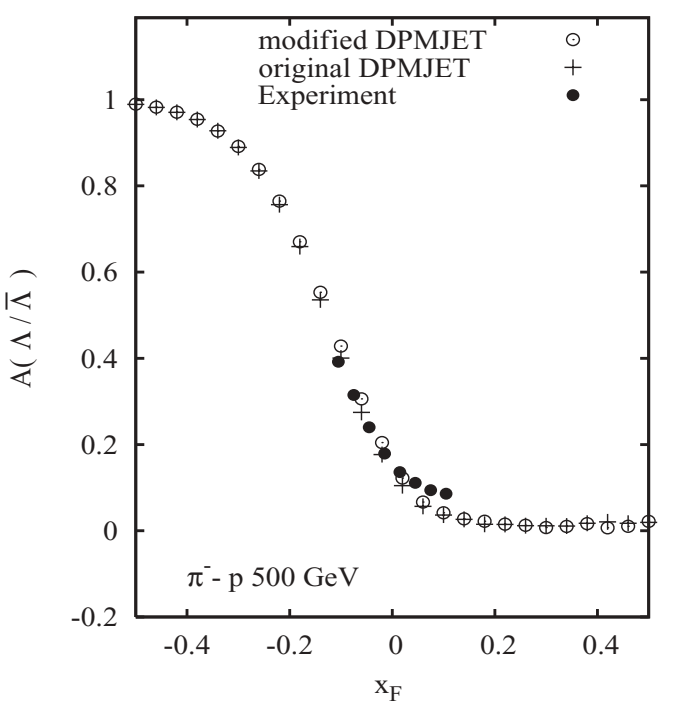

FIG. 15. The $A(\Lambda / \bar{\Lambda})$ asymmety. Plotted are the original DPMJET-III, the modified DPMJET-III, and the experimental data from the E791 Collaboration [9].

In Fig. 15 we present the result for the $A(\Lambda / \bar{\Lambda})$ asymmety. We do not expect that the mechanism according to Fig. 14 changes the $\Lambda$ or $\bar{\Lambda}$ production in an essential way. Indeed, we find in Fig. 15 that the original DPMJET-III and the changed model agree perfectly with each other as well as with the data from the E791 Collaboration [9].

The $A(\Xi / \bar{\Xi})$ and $A(\Omega / \bar{\Omega})$ asymmetries are significantly modified by the mechanism according to Fig. 14 . We plot both asymmetries in Figs. 16 and 17. Again the asymmeties according to the original and modified DPMJET-III are compared to the data from the E791 Collaboration [9]. With the original DPMJET-III the asymmety $A(\Xi / \bar{\Xi})$ is zero in the central region and the asymmetry $A(\Omega / \bar{\Omega})$ is even negative in the central region. With the modified DPMJET-III both

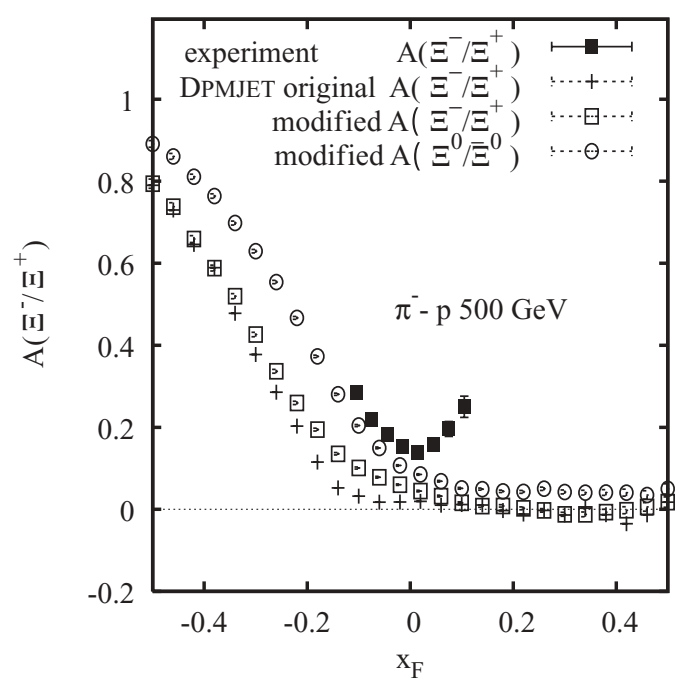

FIG. 16. The $A(\Xi / \bar{\Xi})$ asymmety. Plotted are the original DPMJETIII, the modified DPMJET-III, and the experimental data from the E791 Collaboration [9].

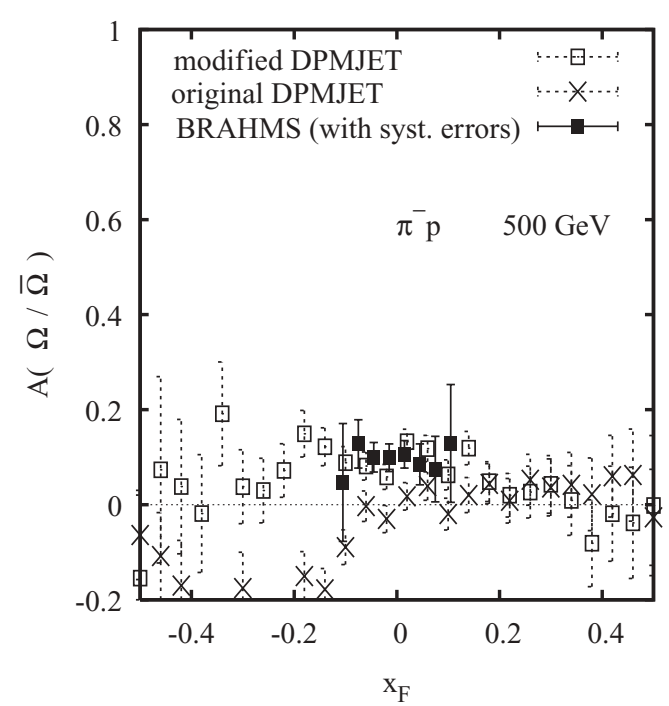

FIG. 17. The $A(\Omega / \bar{\Omega})$ asymmety. Plotted are the original DPMJET-III, the modified DPMJET-III, and the experimental data from the E791 Collaboration [9].

asymmetries become positive in the central region like the experimental data. For the choosen strangeness probability the $\Omega$ asymmetry is somewhat too strong, whereas the $\Xi$ asymmtry is not sufficient.

Most significant seems the rise of of the $\Xi$ asymmetry in the forward region. To investigate the influence of the meson isospin in Fig. 18 the net hyperon contributions are considered for various isospin combinations. For $\pi^{-} p$ the direct influence $\pi^{-} \rightarrow \cdots d \rightarrow \cdots d s s$ rising $\Xi^{-}-\Xi^{+}$but not $\Xi^{0}-\overline{\Xi^{0}}$ seems not to reach the central region where baryons are observed. Obtained is a rank 2 effect $\pi^{-} \rightarrow \cdots \bar{u} \rightarrow \cdots \bar{u} d+\bar{d} \bar{s} \bar{s}$, which, balancing contribution $\pi^{-} \rightarrow \cdots d \rightarrow \cdots \bar{d} d+\bar{d} \bar{s} \bar{s}$, is suppressed by symmetry breaking [as $m(\eta) \gg m\left(\pi^{-}\right)$], yielding $\Xi^{-}-\Xi^{+}$below $\Xi^{0}-\overline{\Xi^{0}}$. The reaction $\pi^{+} p$ has opposite isospin signs.

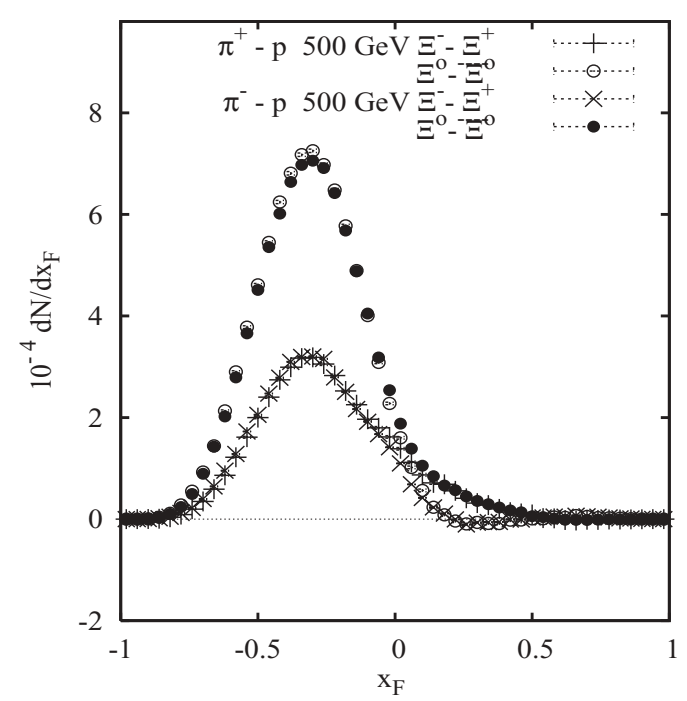

FIG. 18. The net $\Xi-\bar{\Xi}$ contribution for charged an neutral hyperons for $\pi^{+} p$ and $\pi^{-} p$ scattering. 


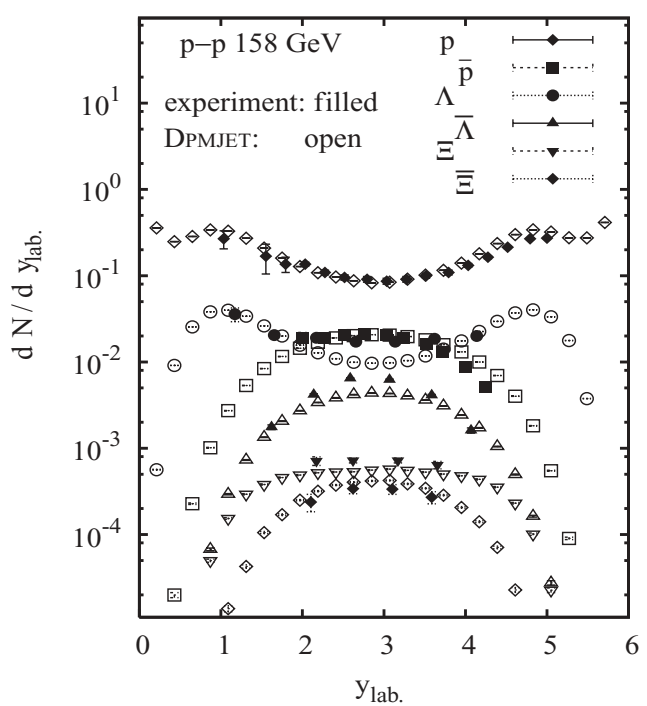

FIG. 19. Rapidity distributions of produced $p, \bar{p}, \Lambda, \bar{\Lambda}, \Xi$, and $\bar{\Xi}$ in proton-proton collisions at $158 \mathrm{GeV}$. We compare the rapidity distributions according to the modified DPMJET-III with experimental data from the NA49 Collaboration [10].

In conclusion the $\pi^{-}$isospin effect is not only too small to explain the effect seen in the data but also it actually contributes in the wrong direction. The error bars are significant. If the rise is confirmed by a second experiment, again, a new effect will have to added. It could provide evidence for the backward peak postulated in Ref. [38] caused by a tiny three chain complete forward-backward exchange contribution leaving a pion and possibly a $s s d$ on the opposite side.

Rapidity distributions of $p, \bar{p}, \Lambda, \bar{\Lambda}, \Xi$, and $\bar{\Xi}$ were measured in proton-proton collisions at $158 \mathrm{GeV}$ by the NA49 Collaboration [10]. Also in this experiment the $\bar{\Xi} / \Xi$ ratio in the central region is found to be $0.44 \pm 0.08$, which is smaller than 1. In the original DPMJET-III as well as in PYTHIA this ratio is found to be approximately equal to 1 . The reasons for this are exactly the same as discussed above. To get better agreement to the data, we had to modify DPMJET-III in the same way as described above, that is, we had to include the mechanism according to Fig. 14.

In Fig. 19 we compare the results of the modified DPMJET-III (using $B_{2 \text { Mesons }}=0.02$ und $r_{s}=30 \%$ ) with the data from the NA49 Collaboration [10]. We find excellent agreements for $p, \bar{p}, \Lambda$, and $\bar{\Lambda}$ production and improved agreements for $\Xi$ and $\overline{\bar{\Xi}}$ production.

\section{SUMMARY}

Hadronic production models like DPMJET-III greatly benefit from constant interaction with new experiments to check the basic premises and allow for refinements. Of particular importance in this respect are data on hadron production in hadron-hadron collisions and $d$-Au collisions measured at RHIC where string interactions are still moderate. In these not-too-dense regions (hadron-hadron collisions and nuclear collisions involving light nuclei) the general features of the model can be considered quite reliable.

For such collisions we found (partly in previous articles [17-19]) five important corrections to be applied to DPMJET-III:

(i) Percolation and fusion of chains; the data from RHIC allow determination of the amount of percolation to be implemented into DPMJET-III; see Refs. [17,19].

(ii) Collision scaling of large $p_{\perp}$ hadron production in $d$-Au collisions; see Ref. [6].

(iii) Replacing the Gaussian transverse-momentum distribution contained in the JETSET/PYTHIA code $[33,34]$ by an exponential distribution in soft hadronic collisions; see Ref. [23].

(iv) Implementation of new diagrams for an improved description of baryon stopping; see Figs. 2 and 4.

(v) Addition of a baryonium-like dimension to the fragmentation of diquark chains to decouple flavor; see Fig. 14.

These corrections are somewhat technical, and they do not involve the basic structure of the string model. The modifications (i) and (iv)-(v) were described above; (ii) and (iii) are not directly related to the question considered here.

In string models the baryon quantum number are carried by vortex lines that play a special role in the string structure. The understanding of baryon and, in particular, the net baryon production is therefore of central importance.

In DPMJET-III, baryon-stopping diagrams have to be included to get a consistent description of the considered RHIC data. With the new RHIC data, supporting anomalous baryon stopping, this is no longer a merely theoretical exercise. Good agreement with the critical experimental data was obtained.
[1] A. Capella, U. Sukhatme, C. I. Tan, and J. Trân Thanh Vân, Phys. Rep. 236, 227 (1994).

[2] R. Engel, Z. Phys. C 66, 203 (1995).

[3] R. Engel and J. Ranft, Phys. Rev. D 54, 4244 (1996).

[4] S. Roesler, R. Engel, and J. Ranft, Copernicus Ges. 1, (2001).

[5] S. Roesler, R. Engel, and J. Ranft, Advanced Monte Carlo for radiation physics, particle transport simulation and applications: Proceedings of Monte Carlo 2000, Lisboa, Oct. 2000 (Springer, New York, 2001), p. 1033.

[6] F. W. Bopp, J. Ranft, R. Engel, and S. Roesler, J. Phys. G. 30 (2004).

[7] T. Alber et al. (NA35 Collaboration), Z. Phys. C 64, 195 (1994).
[8] T. Alber et al. (NA35 Collaboration), Eur. Phys. J. C 2, 643 (1998).

[9] E. M. Aitala et al. (E791 Collaboration), Phys. Lett. B469, 9 (2000).

[10] T. Susa et al. (NA49 Collaboration), Nucl. Phys. A698, 491c (2002).

[11] D. Kharzeev, Phys. Lett. B378, 238 (1996).

[12] A. Capella and B. Kopeliovich, Phys. Lett. B381, 325 (1996).

[13] A. Capella, E. G. Ferreiro, and C. A. Salgado, Phys. Lett. B459, 27 (1999).

[14] A. Capella and C. A. Salgado, Phys. Rev. C 60, 054906 (1999).

[15] S. E. Vance and M. Gyulassy, Phys. Rev. Lett. 83, 1735 (1999). 
[16] J. A. Casado, Nucl. Phys. A651, 93 (1999).

[17] J. Ranft, [hep-ph/0002137] (unpublished).

[18] J. Ranft, R. Engel, and S. Roesler, Copernicus Ges. 435 (2001).

[19] J. Ranft, R. Engel, and S. Roesler, Proceedings of Monte Carlo 2000, Lisboa, Oct. 2000 (Springer, New York, 2000), p. 979.

[20] M. A. Braun, C. Pajares, and J. Ranft, Int. J. Mod. Phys. A 14, 2689 (1999).

[21] M. Braun and C. Pajares, Eur. Phys. J. C 16, 349 (2000).

[22] J. Ranft, R. Engel, and S. Roesler, Nucl. Phys. B, Proc. Suppl. 122, 292 (2003).

[23] F. W. Bopp, J. Ranft, R. Engel, and S. Roesler, QCD at Cosmic Energies, Erice by INFN Eloisatron Project 44th Workshop on QCD at Cosmic Energies, Erice, Sept. 2004.

[24] B. B. Back et al. (PHOBOS Colaboration), Phys. Rev. C 70, 011901 (2004).

[25] P. Steinberg et al. (PHOBOS Collaboration), J. Phys. G: Nucl. Part. Phys. 30, S683 (2004) .

[26] F. Simon (STAR Colaboration), Acta Phys. Hung. A 27, 287 (2006).

[27] I. G. Bearden et al. (BRAHMS Collaboration), Phys. Lett. B607, 42 (2005).

[28] G. C. Rossi and G. Veneziano, Nucl. Phys. B123, 507 (1977).

[29] U. Sukhatme, K. Lassila, and R. Orava, Phys. Rev. D 25, 2975 (1982).

[30] B. Z. Kopeliovich and B. G. Zakharov, Z. Phys. C 43, 241 (1989).
[31] J. Ranft and S. Ritter, Acta Phys. Pol. B 11, 259 (1980).

[32] S. Ritter, Comput. Phys. Commun. 31, 393 (1984).

[33] T. Sjöstrand, Comput. Phys. Commun. 82, 74 (1994).

[34] B. Andersson, G. Gustafson, and T. Sjöstrand, Phys. Scr. 32, 574 (1985).

[35] A. Capella, E. G. Ferreiro, and C. A. Salgado, Nucl. Phys. A661, 502 (1999).

[36] F. W. Bopp and Yu. M. Shabelski, Phys. At. Nucl. 68, 2093 (2005) [hep-ph/0406158]; F. W. Bopp and Yu. M. Shabelski, Eur. Phys. J. A 28, 237 (2006).

[37] P. Aurenche and F. W. Bopp, Nucl. Phys. B119, 157 (1977).

[38] F. W. Bopp, [hep-ph/0002190] (unpublished).

[39] B. Alper et al., Nucl. Phys. B100, 237 (1975).

[40] M. Aguilar-Benitez et al., Z. Phys. C 50, 405 (1991).

[41] G. H. Arakelian, A. Capella, A. B. Kaidalov, and Y. M. Shabelski, Eur. Phys. J. C 26, 81 (2002).

[42] J. C. Dunlop for the STAR Collaboration, Nucl. Phys. A774, 139 (2006).

[43] J. Adams et al. (STAR Collaboration), Phys. Lett. B637, 161 (2006).

[44] A. Capella, Phys. Lett. B542, 65 (2002).

[45] F. M. Liu, J. Aichelin, M. Bleicher, H. J. Drescher, S. Ostapchenko, T. Pierog, and K. Werner, Phys. Rev. D 67, 034011 (2003). 